\title{
Humeral shaft fractures: national trends in management
}

\author{
Bradley S. Schoch ${ }^{1}$ (1) Eric M. Padegimas ${ }^{2} \cdot$ Mitchell Maltenfort $^{3}$. \\ James Krieg ${ }^{3}$ Surena Namdari ${ }^{3}$
}

Received: 2 November 2016/ Accepted: 14 April 2017/Published online: 8 May 2017

(c) The Author(s) 2017. This article is an open access publication

\begin{abstract}
Background The incidence of humeral shaft fractures has been increasing over time. This represents a growing public health concern in a climate of cost containment. The purpose of this study is to analyze national trends in surgical management of humeral shaft fractures and determine factors predictive of surgical intervention.

Materials and methods Humeral shaft fractures were identified by the International Classification of Diseases, Ninth Revision, Clinical Modification codes 812.21 and 812.31 in the United States Nationwide Inpatient Sample from 2002 to 2011. Open reduction and internal fixation (ORIF) was identified by code 79.31 (ORIF, humerus). Other case codes analyzed were 79.01 (closed reduction without internal fixation), 79.11 (closed reduction with internal fixation), and 79.21 (open reduction without internal fixation). Multivariate regression analysis was utilized to determine predictive factors for utilization of ORIF.

Results 27,908 humeral shaft fractures were identified. Utilization of ORIF increased from $47.2 \%$ of humeral shaft fractures in 2002 to $60.3 \%$ in 2011. Demographically, patients who underwent ORIF were younger (51.5 versus 59.7 years, $p<0.001$; odds ratio 0.87 per decade of age). There were modest increases in ORIF usage with private
\end{abstract}

Surena Namdari

surena.namdari@rothmaninstitute.com

1 Department of Orthopaedics and Rehabilitation, University of Florida, Gainesville, FL, USA

2 Department of Orthopedics, Thomas Jefferson University, Philadelphia, PA, USA

3 Department of Orthopaedic Surgery, Shoulder and Elbow Surgery, Rothman Institute, Thomas Jefferson University, 925 Chestnut St, 5th Floor, Philadelphia, PA 19107, USA insurance, open fracture, and hospital size, which persisted with multivariate regression analysis. Surprisingly, there was a tendency to shift from a slight increase in ORIF for males with the bivariate case to a slight preference for females in the multivariate case.

Conclusion Utilization of ORIF for humeral shaft fractures has been steadily increasing with time. Surgical intervention was more common with younger patients, female gender, private insurance, and larger hospital size. The increasing incidence of surgical management for humeral shaft fractures may represent a public health burden given the historical success of non-operative management.

Level of evidence IV.

Keywords Nationwide inpatient sample · NIS · Humerus fracture $\cdot$ Non-operative $\cdot$ Open reduction internal fixation

\section{Introduction}

Humeral shaft fractures represent $3 \%$ of all managed fractures and occur with an incidence of 13 per 100,000 per year $[1,2]$. The incidence of these fractures has been increasing with the aging population [3]. These injuries occur in a bimodal age distribution affecting both young and old patients. Most patients are elderly ( $>65$ years old), representing fragility-type fractures; however, these injuries also occur in younger patients $(<30$ years old) secondary to high-energy trauma [3]. Historically, nonoperative management has been the preferred method for treating humeral shaft fractures, given the shoulder's ability to compensate for angular and rotational malalignment $[4,5]$. Sarmiento popularized non-operative management with a functional brace in 1977 after swelling had abated following 1-2 weeks in a coaptation splint [6, 7]. In 
contrast, both compression plating and intramedullary nailing were developed in an attempt to improve functional outcomes.

Recently, open reduction and internal fixation (ORIF) has become more prevalent, with analysis from Finland finding a two-fold increase in operative management between 1987 and 2009 [8]. However, in the United States, it is unclear how the incidence of ORIF has changed over time. Analysis of national trends may provide insight into changing surgeon and patient expectations. Additionally, understanding the national trends in management of humeral shaft fractures may clarify the public health burden that these injuries might represent. The purpose of this study is to analyze national trends in surgical management of humeral shaft fractures and determine any factors predictive of surgical intervention.

\section{Materials and methods}

This study was conducted in the United States using the Nationwide Inpatient Sample (NIS). All humeral shaft fractures treated between 2002 and 2011 were identified using International Classification of Diseases, Ninth Revision, Clinical Modification (ICD-9-CM) codes. Patients were included if they received a diagnosis of 812.21 (closed fracture of humeral shaft) or 812.31 (open fracture of humeral shaft). The group was then subdivided into those treated non-operatively and those treated operatively. Fractures treated operatively were identified by ICD-9-CM code 79.01 (closed reduction without internal fixation), 79.11 (closed reduction with internal fixation), 79.21 (open reduction without internal fixation), or 79.31 (ORIF). Patients under the age of 18 were excluded, given the possible presence of open physes.

The NIS is currently the largest national payer database with the ability to follow inpatient hospitalizations. Information is captured from $\sim 8$ million patients and encompasses over 1000 hospitals, with results added on a yearly basis. Both federal and private hospitals are available for analysis, including smaller specialty hospitals. The annual survey has been estimated to represent $20 \%$ of all hospital discharges within the United States [9]. The Healthcare Cost and Utilization Project produces yearly statistical analyses to adjust for yearly variation in the NIS sampling $[10,11]$. From this sample, demographic factors of age, gender, and ethnicity were recorded for each patient. Insurance variables and hospital size were also characterized.

Trends in utilization of ORIF over time were analyzed by Pearson's correlation analysis. Multivariate analysis was utilized to determine predictive factors for utilization of ORIF. Microsoft Excel (2013; Redmond, WA, USA) and R [R Development Core Team (2008) R: a language and environment for statistical computing. R Foundation for Statistical Computing, Vienna, Austria] was used for all statistical calculations. Statistical significance was considered for a $p$ value less than 0.05 .

\section{Results}

Between 2002 and 2011, 27,908 humeral shaft fractures were identified through the NIS database. Using the assumption that the NIS database represents $20 \%$ of inpatient hospitalization discharges, Healthcare Cost and Utilization Project (HCUP) statistical corrections were used to estimate a national burden of 149,300 humeral shaft fractures over that time period $[10,11]$. Of the 27,908 unique fractures identified in the sample, there were 15,142 (49.6\%) who underwent ORIF, 4036 (14.5\%) who underwent a surgical procedure other than ORIF (closed reduction without internal fixation, closed reduction with internal fixation, or open reduction without internal fixation), and 8730 (31.3\%) who did not undergo surgical treatment. There were 2672 (9.6\%) open fractures compared to 25,236 (90.4\%) closed. In 2002, there were 2486 (8.9\% of total) with $1174(47.2 \%)$ treated by ORIF, $308(12.4 \%)$ treated with a different surgical procedure, and 1004 (40.4\%) treated non-operatively. By 2011, there were $3033(10.9 \%)$ with $1828(60.3 \%)$ treated by ORIF, $246(8.1 \%)$ treated with a different surgical procedure, and 959 (31.6\%) treated non-operatively. Logistic regression estimated that the probability for a humeral fracture patient receiving ORIF increased annually with an odds ratio (OR) of 1.07 per year [95\% confidence interval (CI) 1.06-1.08], and that the odds for an operatively treated patient receiving ORIF increased with an OR of 1.10 per year (95\% CI 1.08-1.11). The yearly trend for this is shown in Figs. 1 and 2.

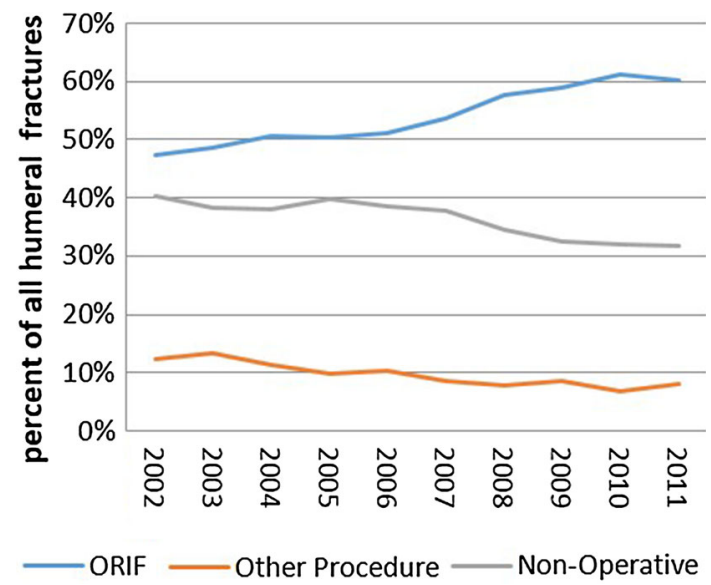

Fig. 1 Annual trend of utilization of all treatment modalities for humeral shaft fractures 


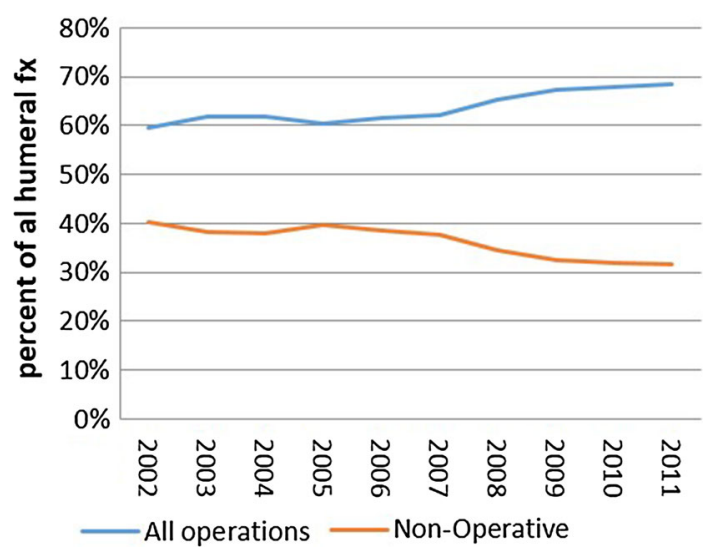

Fig. 2 Annual trend of operative and non-operative management of humeral shaft fractures

Analyzing the entire study population of fracture, patients who underwent ORIF were younger (Fig. 3; 53.2 versus 61.2 years, $p<0.001$ ), as seen in the trend in Fig. 3. Multivariate analysis adjusting for other factors showed odds of ORIF decreased with an OR of 0.87 (95\% CI 0.85-0.88) per decade of age. When gender was analyzed in this multivariate regression analysis, males were slightly more likely to receive ORIF: $54.0 \%$ of males received ORIF versus $49.4 \%$ of females $(p<0.001)$. However, the multivariate analysis showed a slight preference for females getting ORIF (OR of $1.07,95 \%$ CI $1.01-1.13, p=0.026$ ). With respect to insurance type, $42.2 \%$ of national government insurance for patients $>65$ years old (Medicare) and $55.0 \%$ of state government insurance for low-income patients (Medicaid) underwent ORIF compared to $59.4 \%$ of patients with private insurance. Comparing between insurance groups and correcting for multiple comparisons with the Holm-Bonferroni adjustment, Medicare had lower rates of ORIF than any other insurance group $(p<0.001)$, while private insurance had higher rates of ORIF than for self-pay (54.7\%). Open fractures had higher rates of ORIF than closed fractures (65.1 vs $49.9 \%$; adjusted OR $1.47,95 \%$ CI $1.35-2.61$, $p<0.0001)$. ORIF use also increased with the size of the hospital: $44.6 \%$ for small hospitals, $50.4 \%$ for medium, and $52.7 \%$ for large $(p<0.0001)$.

\section{Discussion}

Union rates with non-operatively treated humeral shaft fracture have been reported between 67 and 98\% [12-14]. Despite these rates, some patients are unable or unwilling to undergo non-operative management. Clinical union and removal of brace takes an average of 11.5 weeks with a range of 4-22 weeks with functional bracing compared with 6.3-9.8 weeks for intramedullary nailing and 8.9-10.4 weeks for compression plating [14-16]. Return to weight-bearing remains a function of bone quality and surgical fixation. Weight-bearing restrictions may be devastating to the elderly, who often require their arm to transfer or even weight-bear. In the younger patient, nonoperative management may also delay their ability to return to work. In addition to functional limitations, functional bracing also carries a $1-9.5 \%$ risk of skin and soft tissue complications [17-19].

We demonstrate that surgical treatment of humeral shaft fractures in the United States has been increasing over time. The reason for this rise remains unclear, as numerous studies have reported satisfactory treatment with non-operative management. Complications following ORIF also occur at a similar rate to bracing, albeit with a different profile. Nerve palsy is the most common complication, reported in up to $7 \%$ of patients. Infection is also a common complication, affecting up to $3 \%$ of patients [20]. Possible reasons for increased ORIF utilization include a perceived quicker return to work, earlier initiation of shoulder and elbow rehabilitation, and avoidance of brace wear during the recovery period. While fixed-angle locked plating was introduced in 2005 and has been described for comminuted humeral shaft fractures and osteoporotic bone, the mainstay of treatment remains non-locked plating
Fig. 3 Utilization of operative intervention for humeral shaft fractures by age

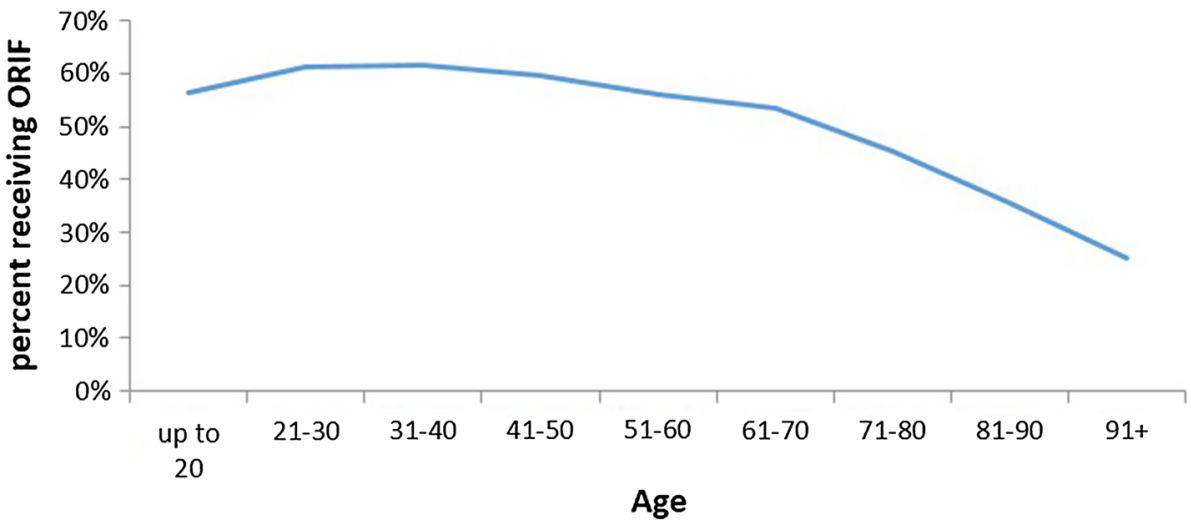


$[21,22]$. Additionally, the increase in ORIF seen over time predated the introduction of locked plating, with no upward inflexion point seen over time. As a result, we do not believe that advancements in plate technology are responsible for the increased ORIF utilization described in this study. However, the development of intramedullary nailing for humeral shaft fractures does coincide with the timing of the increase in operative intervention [23, 24]. The increasing utilization of this technique may correlate with the observed trend.

Multiple predictive factors for operative intervention were identified. The first was younger age. We are unable to analyze other potential confounding factors which would make fixation more common in younger patients. However, older patients with osteoporotic bone and more medical comorbidities may be less willing to undertake or less likely to be recommended for surgery. Younger patients are also more likely to be involve in high energy poly-trauma [25], which is a relative indication for fixation of a humeral shaft fracture [26, 27]. Younger patients also have more demands to return to work and may not accept a longer duration of functional limitations. This result is in contrast with that of Matuszeqski et al. who showed that patients undergoing surgical fixation of humeral shaft fractures in the United States National Trauma Database were 3.5 years older than those treated non-operatively [28].

Open fractures were highly associated with fixation, which is a well-established indication for surgical intervention [23]. Patients with private insurance were also more likely to undergo operative intervention. This was an independent association on multivariate regression analysis and therefore not simply driven by Medicare patients being statistically older than privately insured patients. This finding is similar to a previous analysis that identified an association between surgery for upper extremity fracture and private insurance [29]. Additionally, previous analysis of supracondylar humerus fractures treated as outpatients in the pediatric population found that privately insured patients were nearly two and a half times as likely to return for surgical intervention than those with public or no insurance [30]. This independent association between lower surgical intervention rates and non-private insurance may be a barrier to care that pushes the treating surgeon to admit these patients rather than discharge for outpatient follow-up.

This study has multiple limitations. While the NIS database is a validated tool for studying population-based usage of medical resources, it remains limited by its capture of $20 \%$ of the population and relies on statistical modifiers to extrapolate to national projections. Additionally, as the NIS is an inpatient database, it is only able to capture those fractures that were admitted. Therefore, any patient treated in the emergency department with a coaptation splint, discharged for outpatient follow-up, and treated non-operatively would be missed. Similarly, any patient discharged for outpatient follow-up treated operatively, but in an outpatient surgical setting in which they were not admitted, would be missed. This study also does not explore the relationship between surgical fixation and discharge disposition, a relationship described by Matuszewski et al. [28]. Lastly, the NIS does not capture fracture classification or fixation type (plate size, locking/nonlocking), making analysis based on these variables impossible. Further outcomes-based studies are needed to identify the effect of increased operative fixation on patient outcomes in these groups.

In conclusion, humeral shaft fractures have continued to increase along with an aging population. Independent of this increased prevalence of humeral shaft fractures, the utilization of ORIF in the United States has also trended upwards in a similar manner. Predictive factors for operative ultization were identified as younger age, open fracture, and private insurance.

\section{Compliance with ethical standards}

Conflict of interest Bradley Schoch is a paid speaker for DJO. James Krieg is a paid consultant for Conventus, Merck, and Synthes. James Krieg owns stock in Conventus, Domain Surgical, MDLive, and Trice Medical. James Krieg receives royalties from Synthes and SAM Medical. Surena Namdari receives divisional research funding from Depuy, Zimmer, Integra Life Sciences, and Arthrex. Surena Namdari is a paid consultant for DJO Surgical, Integra Life Sciences, and Miami Device Solutions. Surena Namdari receives royalty payments from DJO Surgical, Miami Device Solutions, and Elsevier. The authors declare that they have no conflict of interest in relation to this manuscript.

Patient consent Unnecessary, since data is obtained from a public data base of anonymized patients.

Ethical approval Unnecessary, since data is obtained from a public data base of anonymized patients.

Funding No external funding was received for this study.

Open Access This article is distributed under the terms of the Creative Commons Attribution 4.0 International License (http://crea tivecommons.org/licenses/by/4.0/), which permits unrestricted use, distribution, and reproduction in any medium, provided you give appropriate credit to the original author(s) and the source, provide a link to the Creative Commons license, and indicate if changes were made.

\section{References}

1. Court-Brown CM, Caesar B (2006) Epidemiology of adult fractures: a review. Injury 37:691-697. doi:10.1016/j.injury.2006.04.130

2. Ekholm R, Adami J, Tidermark J et al (2006) Fractures of the shaft of the humerus. An epidemiological study of 401 fractures. J Bone Joint Surg Br 88:1469-1473. doi:10.1302/0301-620X. 88B11.17634 
3. Kim SH, Szabo RM, Marder RA (2012) Epidemiology of humerus fractures in the United States: nationwide emergency department sample, 2008. Arthritis Care Res 64:407-414. doi:10. 1002/acr.21563

4. Holm CL (1970) Management of humeral shaft fractures. Fundamental nonoperative technics. Clin Orthop 71:132-139

5. Klenerman L (1966) Fractures of the shaft of the humerus. J Bone Joint Surg Br 48:105-111

6. Sarmiento A, Kinman PB, Galvin EG et al (1977) Functional bracing of fractures of the shaft of the humerus. J Bone Joint Surg Am 59:596-601

7. Walker M, Palumbo B, Badman B et al (2011) Humeral shaft fractures: a review. J Shoulder Elbow Surg 20:833-844. doi:10. 1016/j.jse.2010.11.030

8. Huttunen TT, Kannus P, Lepola V et al (2012) Surgical treatment of humeral-shaft fractures: a register-based study in Finland between 1987 and 2009. Injury 43:1704-1708. doi:10.1016/j. injury.2012.06.011

9. Healthcare Cost and Utilization Project (HCUP) (2014) Introduction to the HCUP National Inpatient Sample (NIS) 2012. Agency for Healthcare Research and Quality, Rockville

10. Houchens R, Elixhauser A (2014) Using the HCUP Nationwide Inpatient Sample to estimate trends (updated for 1988-2004). https://www.hcup-us.ahrq.gov/reports/methods/methods.jsp. Accessed March 5th, 2016

11. Kurtz SM, Lau E, Ong K et al (2009) Future young patient demand for primary and revision joint replacement: national projections from 2010 to 2030. Clin Orthop 467:2606-2612. doi:10.1007/s11999-009-0834-6

12. Ali E, Griffiths D, Obi N et al (2015) Nonoperative treatment of humeral shaft fractures revisited. J Shoulder Elbow Surg 24:210-214. doi:10.1016/j.jse.2014.05.009

13. Denard A, Richards JE, Obremskey WT et al (2010) Outcome of nonoperative vs operative treatment of humeral shaft fractures: a retrospective study of 213 patients. Orthopedics. doi:10.3928/ 01477447-20100625-16

14. Sarmiento A, Zagorski JB, Zych GA et al (2000) Functional bracing for the treatment of fractures of the humeral diaphysis. J Bone Joint Surg Am 82:478-486

15. Changulani M, Jain UK, Keswani T (2007) Comparison of the use of the humerus intramedullary nail and dynamic compression plate for the management of diaphyseal fractures of the humerus. A randomised controlled study. Int Orthop 31:391-395. doi:10. 1007/s00264-006-0200-1

16. Chapman JR, Henley MB, Agel J, Benca PJ (2000) Randomized prospective study of humeral shaft fracture fixation: intramedullary nails versus plates. J Orthop Trauma 14:162-166

17. Jawa A, McCarty P, Doornberg J et al (2006) Extra-articular distal-third diaphyseal fractures of the humerus. A comparison of functional bracing and plate fixation. J Bone Joint Surg Am 88:2343-2347. doi:10.2106/JBJS.F.00334

18. Koch PP, Gross DFL, Gerber C (2002) The results of functional (Sarmiento) bracing of humeral shaft fractures. J Shoulder Elbow Surg 11:143-150

19. Woon CY-L (2010) Cutaneous complications of functional bracing of the humerus: a case report and literature review. J Bone Joint Surg Am 92:1786-1789. doi:10.2106/JBJS.I.01309

20. Gottschalk MB, Carpenter W, Hiza E et al (2016) Humeral shaft fracture fixation: incidence rates and complications as reported by american board of orthopaedic surgery part ii candidates. J Bone Joint Surg Am 98:e71. doi:10.2106/JBJS.15.01049

21. Gardner MJ, Griffith MH, Demetrakopoulos D et al (2006) Hybrid locked plating of osteoporotic fractures of the humerus. J Bone Joint Surg Am 88:1962-1967. doi:10.2106/JBJS.E.00893

22. Dai J, Chai Y, Wang C, Wen G (2014) Dynamic compression plating versus locked intramedullary nailing for humeral shaft fractures: a meta-analysis of RCTs and nonrandomized studies. J Orthop Sci 19:282-291. doi:10.1007/s00776-013-0497-8

23. Carroll EA, Schweppe M, Langfitt M et al (2012) Management of humeral shaft fractures. J Am Acad Orthop Surg 20:423-433. doi:10.5435/JAAOS-20-07-423

24. Stannard JP, Harris HW, McGwin G et al (2003) Intramedullary nailing of humeral shaft fractures with a locking flexible nail. J Bone Joint Surg Am 85:2103-2110

25. Bardenheuer M, Obertacke U, Waydhas C, Nast-Kolb D (2000) Epidemiology of the severely injured patient. A prospective assessment of preclinical and clinical management. AG Polytrauma of DGU. Unfallchirurg 103:355-363

26. Blum J, Gercek E, Hansen M, Rommens PM (2005) Operative strategies in the treatment of upper limb fractures in polytraumatized patients. Unfallchirurg 108(843-844):846-849. doi:10. 1007/s00113-005-1003-3

27. Wali MGR, Baba AN, Latoo IA et al (2014) Internal fixation of shaft humerus fractures by dynamic compression plate or interlocking intramedullary nail: a prospective, randomised study. Strateg Trauma Limb Reconstr Online 9:133-140. doi:10.1007/s11751-014-0204-0

28. Matuszewski PE, Kim TW, Gay AN, Mehta S (2015) Acute operative management of humeral shaft fractures: analysis of the national trauma data bank. Orthopedics 38:e485-e489. doi:10. 3928/01477447-20150603-56

29. Patel AA, Buller LT, Fleming ME et al (2015) National trends in ambulatory surgery for upper extremity fractures: a 10-year analysis of the US National Survey of Ambulatory Surgery. Hand (N Y) 10:254-259. doi:10.1007/s11552-014-9703-1

30. Fletcher ND, Sirmon BJ, Mansour AS et al (2016) Impact of insurance status on ability to return for outpatient management of pediatric supracondylar humerus fractures. J Child Orthop. doi:10.1007/s11832-016-0769-x 